\title{
Validação e Confiabilidade de um Instrumento de Avaliação de Feridas
}

\author{
Validity and Reliability of an Instrument for Wound Evaluation \\ Validación y Confiabilidad de un Instrumento de Evaluación de Heridas
}

\section{Helena Maria Bajay', Izilda Esmenia Muglia Araújo²}

\section{RESUMO}

Objetivo: este estudo teve como objetivos validar e verificar a confiabilidade de um instrumento elaborado para a avaliação de feridas. Métodos: o estudo foi desenvolvido num hospital universitário. A validade do instrumento foi apreciada por juízes e para avaliar a confiabilidade foi realizada a análise de concordância entre observadores em relação aos dados da ferida. Resultados: indicam que os juízes compreenderam a maioria dos itens do instrumento com $71 \%$ de respostas positivas e os níveis de concordância entre observadores foram regulares e excelentes. Conclusão: sugere-se a aplicação do instrumento pelos profissionais, pois acredita-se na sua praticidade.

Descritores: Feridas; Confiabilidade; Pesquisa em enfermagem; Estudos de validação

\begin{abstract}
Objective: to determine the reliability and validity of a new instrument for wound evaluation. Methods: this methodological study was conducted in a Brazilian university hospital. Data analysis consisted of content validity by a panel of judges and equivalency among observers. Results: the new instrument had a good validity; the level of agreement among the judges was $71 \%$. And, the interrater reliability and the agreement between observers were regular to excellent. Conclusion: this instrument has the potential to be used by nurses in clinical practice.
\end{abstract}

Keywords: Wound; Reliability; Nursing Research; Validity

\section{RESUMEN}

Objetivo: en este estudio se tuvo como objetivo validar y verificar la confiabilidad de un instrumento elaborado para la evaluación de heridas. Métodos: el estudio fue desarrollado en un hospital universitario. La validación del instrumento fue realizada por jueces y para evaluar la confiabilidad se efectuó el análisis de concordancia entre observadores en relación a los datos de la herida. Resultados: indican que los jueces comprendieron la mayoría de los items del instrumento con el $71 \%$ de respuestas positivas y, los niveles de concordancia entre observadores fueron regulares y excelentes. Conclusión: se sugiere la aplicación del instrumento por los profesionales, pues se cree en su practicidad.

Descriptores: heridas; Confiabilidad; Investigación en enfermería; Estudios de validación

\footnotetext{
${ }^{1}$ Mestre em Enfermagem ; Enfermeira do Serviço de Enfermagem de Emergência Clínica e Cirurgia do Trauma do Hospital das Clinicas, Universidade Estadual de Campinas UNICAMP - Campinas (SP), Brasil.

2 Professor Assistente, Doutor do Departamento de Enfermagem da Faculdade de Ciências Médicas da Universidade Estadual de Campinas - UNICAMP - Campinas (SP), Brasil.
} 


\section{INTRODUÇÃO}

Os enfermeiros exercem importante papel no tratamento das lesões cutâneas e devem refletir sobre a sua prática em busca de novos conhecimentos. Essa prática, ao longo dos anos, passa por profundas transformações, desafiando o conhecimento técnicocientífico dos enfermeiros. Porém, muitas vezes, eles ainda encontram dificuldades para identificar a fase correta da cicatrização e confundem as características normais e anormais associadas a esse processo ${ }^{(1-2)}$.

Avaliar uma ferida pode ocasionar interpretações variadas devido a sua diversidade quanto à natureza, forma e localização, além da percepção própria de cada enfermeiro, tendo em vista a diferença de conhecimentos que existe entre os profissionais que realizam essa prática. Uma mesma ferida pode ser avaliada e ter diferentes registros, podendo gerar interpretações divergentes ou conflitantes. Para garantir a confiança interobservadores, faz-se necessário que o parecer de um profissional coincida com o de seus colegas. Essa confiabilidade pode ser garantida por meio de instrumentos precisos, com padrões e critérios definidos ${ }^{(3)}$. Dentre eles a localização anatômica, tamanho da lesão, cor, tipo de tecido lesado e sua extensão, presença de corpos estranhos, fístulas, túneis e cistos, condição da pele ao redor e característica do exsudato ${ }^{(4-5)}$.

Observando a prática clínica do cuidar de feridas, percebe-se que há deficiências nos registros relativos à descrição das características das feridas. Essas omissões podem levar a equipe médica a retirar os curativos dos pacientes para visualizar a característica da lesão, consumindo material e tempo para refazê-los, além de causar desconforto para o paciente. Ao refletir sobre essa prática, o que se observa é a falta de hábito da equipe de enfermagem de fazer registros mais completos e adequados sobre sua própria atuação.

$\mathrm{Na}$ tentativa de aperfeiçoar a descrição das características da ferida e sua evolução, foi elaborado um instrumento contendo dados da ferida, que possam ser relacionados ao tratamento em curso e aos possíveis fatores de risco que podem interferir na cicatrização. $\mathrm{O}$ impresso proposto deverá ser preenchido pelos enfermeiros ao avaliar a ferida durante a troca dos curativos e permitirá, então, um registro sistemático das características da ferida, com uma visualização global desses dados para análise de sua evolução ao longo do tratamento realizado. Desta forma poderá facilitar a detecção de possíveis complicações, que possam interferir no processo de cicatrização; redirecionar as condutas a fim de eliminar esses fatores e, propiciar condições adequadas de cicatrização. Este instrumento encontra-se divulgado em recente publicação( ${ }^{(6)}$ e o mesmo possui um roteiro para orientar o preenchimento.
O presente estudo é parte da dissertação de mestrado em que se realizou a elaboração, validação e a confiabilidade de um instrumento de registro da evolução.

\section{OBJETIVOS}

Validar e verificar a confiabilidade de um instrumento elaborado para a avaliação de feridas.

\section{MÉTODOS}

O estudo foi desenvolvido nas Unidades de Internação de Emergência Clínica e Cirurgia do Trauma de um hospital universitário, por serem unidades que recebem pacientes graves, cirúrgicos e clínicos, com feridas diversas, no período de outubro a fevereiro de 2001.

Fizeram parte do estudo 31 sujeitos. O tamanho da amostra foi definido com base no número das variáveis presentes no instrumento, sob orientação do Serviço de Estatística da Comissão de Pesquisa da própria instituição. O projeto de pesquisa foi submetido ao Comitê de Ética em Pesquisa da instituição e aprovado sob parecer n¹90/ 2000. A participação na pesquisa foi voluntária, ocorrendo após as orientações, escla-recimentos, autorização e assinatura do Termo de Consentimento Livre Esclarecido, ressaltado o direito à recusa a qualquer momento.

A inclusão de pacientes na amostra foi realizada sem limite de idade, sexo ou raça e com base nos seguintes critérios: a) presença de lesões de pele, com perda de epiderme, detectadas através de inspeção, podendo atingir desde as camadas mais profundas do tecido epitelial, até a exposição de vísceras, músculos, osso, etc. b) lesões de até 40 centímetros, em sua maior extensão (comprimento ou largura).

Foram excluídos da amostra os pacientes com feridas provenientes de inserções de cateteres; drenos; introdutores e fixadores; incisões cirúrgicas fechadas; tumores exteriorizados; lesões sem perda de epiderme; lesões que se localizam em regiões curvas de difícil medição com régua; lesões nos dedos dos pés e das mãos; lesões maiores de $40 \mathrm{~cm}$ de extensão e lesões que se unem por proximidade, lesões dermatológicas, estomias e inserções de drenos.

Validação é um procedimento metodológico em que se avalia a qualidade de um instrumento. A validade deste pode ser relacionada ao contexto em que o mesmo está sendo utilizado e suas variáveis podem ser adaptadas ao referencial teórico adotado ${ }^{(7-8)}$. Para averiguar a validade do instrumento elaborado optou-se em submetê-lo à apreciação de juízes, junto com o seu respectivo roteiro de preenchimento. Foi estabelecido o valor de $70 \%$ para o total de respostas positivas.

A confiabilidade do instrumento foi verificada por 
meio da análise da concordância entre observadores, em relação aos dados da ferida.

A validação de um instrumento pode ser realizada submetendo-o à apreciação de juízes com experiência na área. Esses juízes analisam o conteúdo, a apresentação, a clareza e a compreensão do instrumento ${ }^{(7,9-13)}$.

$\mathrm{O}$ instrumento sugerido, seu respectivo roteiro de preenchimento e o instrumento de avaliação dos juízes foram encaminhados para três juízes, docentes de uma universidade pública do Estado de São Paulo, da área de enfermagem médico-cirúrgica, com domínio do tema, para avaliação quanto à originalidade e criatividade; organização, objetividade e clareza dos itens; itens necessários, porém ausentes no instrumento; itens desnecessários no instrumento e comentários e/ou sugestões.

O método de equivalência ou concordância consiste em dois observadores empregarem o mesmo instrumento para medir os mesmos fenômenos, simultaneamente, registrando de forma independente as informações relevantes ${ }^{(7-9,11)}$.

Para este trabalho, foram considerados relevantes os Dados das Feridas e a escolha dessa parte do instrumento foi em razão da possibilidade de ocorrerem leituras diferentes durante a observação da ferida exposta. Os dados que poderiam ser registrados de forma divergente pelos observadores foram: a classificação da ferida (perda de tecido, graus e profundidade), o tamanho da ferida (extensão maior e menor), a aparência da ferida (granulação, epitelização, fibrina, necrose, outros tipos de tecido, característica ao redor da ferida e sinais flogísticos) e o exsudato (aspecto, quantidade, coloração e odor).

Os sujeitos foram selecionados pelas pesquisadoras, obedecendo aos critérios estabelecidos. As avaliações foram realizadas em dia e hora previamente definidos pelas observadoras, de acordo com a disponibilidade de ambas. A ferida foi observada pelas duas observadoras simultaneamente e sem comunicação entre si até atingir pelo menos duas avaliações consecutivas de cada uma.

Após as observações concomitantes o preenchimento do item Dados das Feridas foi realizado simultaneamente pelas observadoras em separado para testar a confiança das respostas. Os instrumentos foram identificados pelas letras A e B, distinguindo-se assim quem os realizou. A observadora A, uma das autoras da pesquisa, e a observadora $\mathrm{B}$, uma enfermeira com domínio do assunto e que aceitou o convite para a participação na pesquisa. Esta observadora avaliou os dados da ferida no mesmo momento, preenchendo os respectivos campos, de acordo com as orientações contidas no roteiro de preenchimento do instrumento.

Os dados obtidos foram tabulados eletronicamente com auxílio do programa Microsoft EXCEL - 97 e analisados quantitativamente sob orientação do Serviço de Estatística da Comissão de Pesquisa da instituição. O programa de computador utilizado para a análise estatística foi The SAS System for Windows (Statistical Analysis System), versão 6.12. SAS Institute, 1989-1996, NC, USA.

Para a análise do instrumento "Avaliação de Feridas" e do respectivo roteiro de preenchimento, realizada pelos juízes, foi utilizado o coeficiente de concordância Kappa Múltiplo e o teste qui-quadrado de Cochran, para analisar a concordância entre eles. $O$ nível de significância adotado foi de $5 \%(\mathrm{p}<0,05)$.

Para o teste de equivalência das respostas entre os observadores no item Dados das Feridas, utilizou-se o coeficiente de concordância Kappa Simples e esse coeficiente pode ser classificado de diferentes formas, de acordo com os autores. Para este estudo, foi utilizada a escala simplificada de classificação ${ }^{(14)}$, na qual os valores de Kappa abaixo de 0,40 indicam concordância fraca, entre 0,40 e 0,75 indicam concordância regular e acima de 0,75 indicam concordância excelente. $\mathrm{O}$ zero significa ausência de concordância para além do que seja casual, e o um significa concordância perfeita. Também foram consideradas semelhantes as variações de medidas da extensão da ferida quando variaram entre 0,1 e $0,5 \mathrm{~cm}$ e os valores em porcentagem que estiveram entre $1 \% \mathrm{e}$ $10 \%$.

\section{RESULTADOS}

As avaliações do instrumento retornaram às pesquisadoras após dois meses, junto com a análise realizada, os juízes enviaram sugestões de alterações e acréscimos, assim como sugestões de bibliografias.

O coeficiente Kappa Múltiplo indicou ausência de concordância entre eles e o teste qui-quadrado de Cochran obtido $(p<0,001)$ mostrou que houve diferença estatisticamente significativa no número de respostas afirmativas e negativas entre os juízes. Fato ocorrido por que um dos juízes emitiu mais respostas negativas do que os demais. Embora tenha resultado em diferença significativa, o total de respostas positivas continuou maior, indicando que os juízes compreenderam a maioria dos itens do instrumento e que o conteúdo deste poderia ser entendido pelos enfermeiros. Em relação à distribuição das respostas da análise do instrumento, pelos juízes, observou-se que $71,1 \%$ dos itens analisados obtiveram respostas afirmativas, sugerindo que o instrumento foi compreendido e pode ser aperfeiçoado com as sugestões manifestadas por eles e acatadas pelas pesquisadoras.

Os resultados obtidos no teste de concordância entre as observadoras, em relação aos dados das feridas, estão apresentados nas Figuras 1 a 5. 


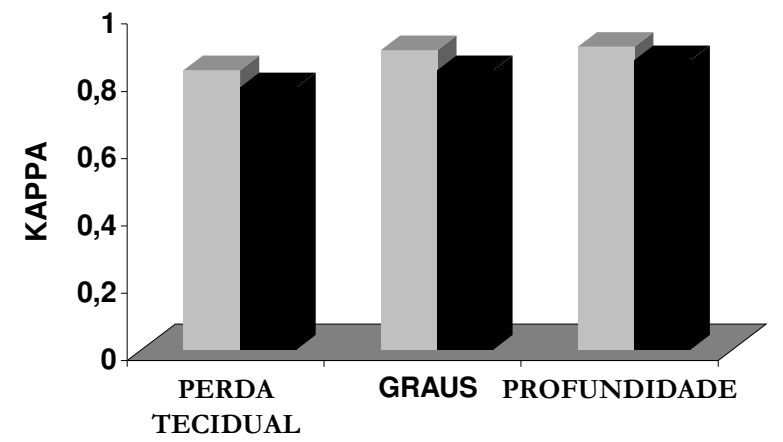

$1^{\mathrm{a}}$ av $\boldsymbol{\sim} 2^{\mathrm{a}}$ av

Figura 1 - Distribuição dos níveis de concordância em relação à classificação das feridas, em perda tecidual, grau e profundidade na $1^{\mathrm{a}}$ e $2^{\mathrm{a}}$ avaliação.

$\mathrm{Na}$ Figura 1 verifica-se que as observadoras apresentaram níveis de concordância elevados (maior $0,75)$ e demonstraram semelhanças entre as classificações.

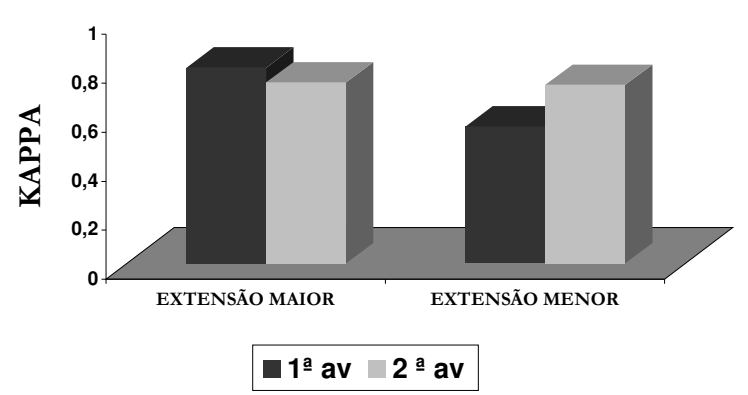

Figura 2 - Distribuição dos níveis de concordância em relação ao tamanho das feridas, em extensão maior e menor na $1^{\mathrm{a}} \mathrm{e} 2^{\mathrm{a}}$ avaliação.

A Figura 2 demonstra que as observadoras apresentaram níveis de concordância elevados (maior que 0,75 ) para ambas as avaliações de extensão maior e na $2^{\mathrm{a}}$ avaliação, da menor extensão, mas para a $1^{\mathrm{a}}$ avaliação da extensão menor o coeficiente obtido foi regular $(0,40$ a 0,75$)$.

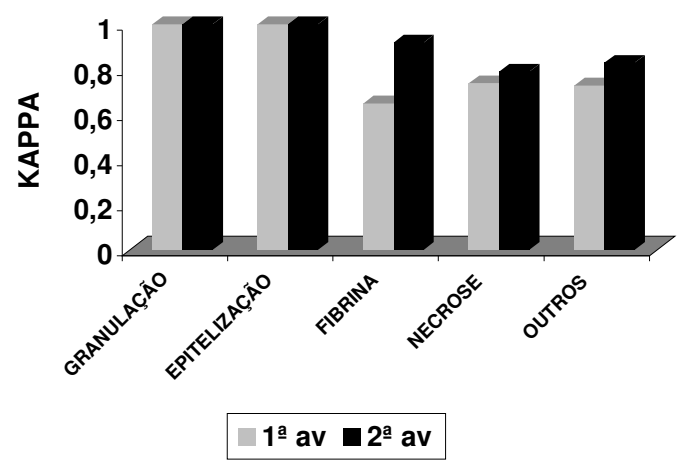

Figura 3 - Distribuição dos níveis de concordância quanto ao tipo de tecido presente no leito da ferida, na $1^{\mathrm{a}}$ e $2^{\mathrm{a}}$ avaliação.

Os resultados apontados na Figura 3 indicam valores de concordância elevados (maior 0,75 ) entre as observadoras, exceto na primeira avaliação em relação à presença de fibrina que apresentou coeficiente regular $(0,40$ a 0,75$)$.

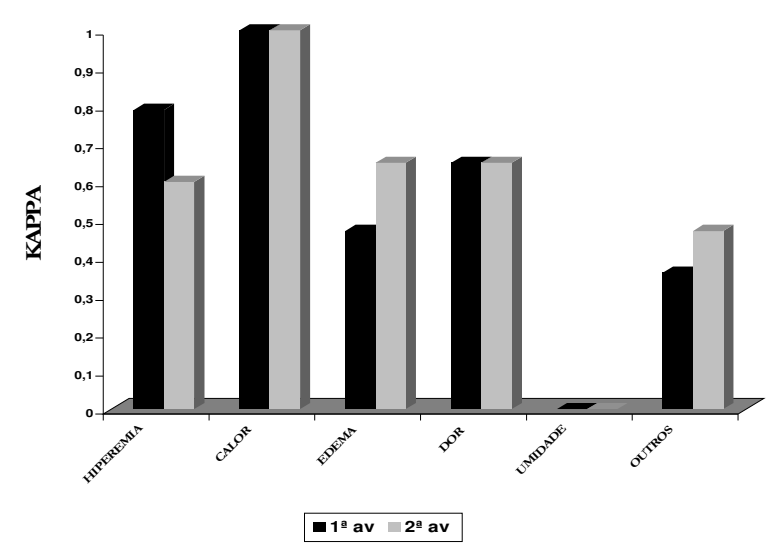

Figura 4 - Distribuição dos níveis de concordância das características da pele ao redor da ferida, na $1^{\mathrm{a}}$ e $2^{\mathrm{a}}$ avaliação.

São apresentados na Figura 4 os resultados dos níveis de concordância das características da pele ao redor da ferida que se mantiveram elevados (maior 0,75 ) na $1^{\text {a }}$ avaliação para hiperemia e calor; regular para edema, dor e outras alterações. Na $2^{a}$ avaliação os níveis foram elevados para calor e, regulares para hiperemia, edema, dor e demais alterações. A umidade não obteve concordância por ter sido detectado somente por um dos avaliadores.

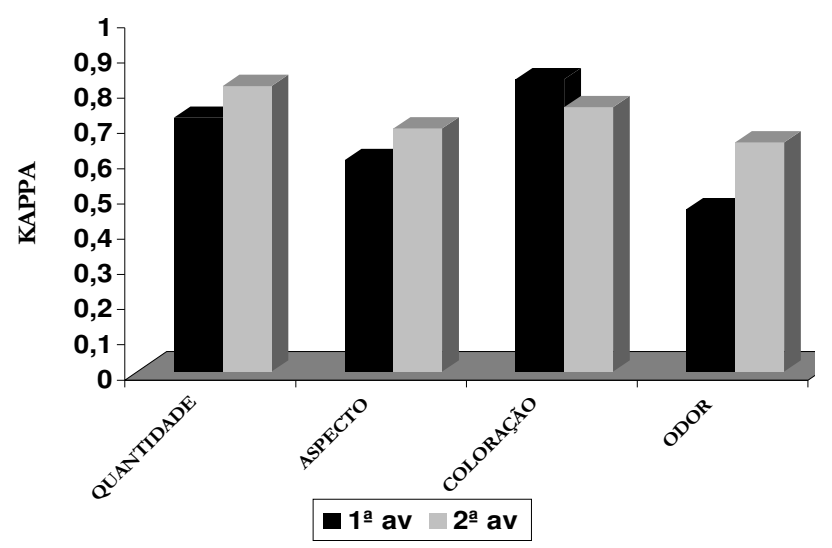

Figura 5 - Distribuição dos níveis de concordância nas características do exsudado das feridas, na $1^{\mathrm{a}}$ e $2^{\mathrm{a}}$ avaliação.

Os resultados presentes na Figura 5 demonstraram níveis regulares de concordância para o aspecto do exsudato e odor nas duas avaliações e, níveis elevados de concordância apenas na $2^{a}$ avaliação da quantidade de exsudato e nas duas avaliações de coloração.

\section{DISCUSSÃO}

Os instrumentos devem ser simples e de fácil 
utilização, e para ser válido ele deve ser utilizado com algum objetivo e prever resultados ${ }^{(3)}$. É enfatizado que para um instrumento ser válido ele deve medir o que se propõe medir ${ }^{(15)}$. No caso de instrumentos de avaliação de feridas é proposta a sua descrição clínica nas diversas fases do processo de cicatrização.

O teste de concordância entre observadores é utilizado para avaliar a confiabilidade de um instrumento. Esta confiabilidade pode ser avaliada pelo grau de coerência e precisão com que um instrumento mede seu atributo. Quanto menor a variação por ele produzida em repetidas mensurações, maior será a sua confiabilidade. Um instrumento é confiável quando suas medidas refletem, de maneira precisa, as medidas do atributo investigado ${ }^{7-}$ 8,11 .

A descrição precisa das características das feridas depende da habilidade do observador em reconhecer as fases do processo de cicatrização e dos fatores que nele podem interferir. A diferença de conhecimento que existe entre os profissionais que realizam essa prática pode ocasionar interpretações variadas ${ }^{(3-4)}$.

A avaliação de uma ferida pode ser realizada por vários sistemas de classificação ${ }^{(16)}$. Para este trabalho, optou-se por utilizar os sistemas de classificações por perda tecidual, grau e profundidade para testá-los e averiguar qual deles apresentava melhor concordância.

Um sistema de classificação pode parecer simples e fácil para se obter os dados, entretanto, uma classificação inadequada do estado da ferida pode ocasionar conseqüências graves, como a utilização indevida de terapias tópicas, desencadeando complicações e retardando a cicatrização ${ }^{(17)}$.

Neste estudo as observadoras apresentaram níveis de concordância elevados e demonstraram semelhanças entre as classificações (Figura 1) podendo qualquer uma delas ser utilizada para classificar as feridas. Entretanto, acreditase que o sistema de classificação por profundidade parece ser mais abrangente que os demais.

O sistema simples de medida é descrito por vários autores como de fácil realização, prático e econômico, apesar de impreciso ${ }^{(1,18-19)}$.

Como optou-se por utilizar a medição simples das feridas na realização desta pesquisa foi necessário estabelecer um critério para posicionar a régua, pois as medições ocasionariam resultados não confiáveis devido ao seu posicionamento aleatório. Mesmo assim os resultados reforçam a necessidade de estabelecer melhor os critérios que determinam os pontos de referências para a medição, pois, as observadoras apresentaram níveis de concordância elevados para ambas as avaliações de extensão maior e na $2^{\mathrm{a}}$ avaliação da menor extensão, mas para a $1^{a}$ avaliação da extensão menor o coeficiente obtido foi regular (Figura 2).

Ao observar as feridas, os enfermeiros devem estar atentos para avaliar além do tamanho, a descrição clínica, pois, apesar da redução de área lesada ser utilizada para julgar a evolução da ferida, às vezes, o aumento de sua extensão após desbridamento pode indicar a melhora da característica da lesão pela remoção do tecido desvitalizado.

A característica do tecido presente no leito da ferida, em porcentagem aproximada, tem sido indicada para descrever sua característica clínica. Os enfermeiros têm importante papel na detecção do tipo de tecido presente na ferida para optar pelo melhor método de tratamento e, dessa forma contribuir para minimizar o tempo de cura. Neste estudo os resultados indicaram valores de concordância elevados entre as observadoras, exceto na primeira avaliação em relação à presença de fibrina que apresentou coeficiente regular. Verifica-se que os enfermeiros podem apresentar percepções distintas da descrição clínica da característica do tecido no leito da ferida. Acredita-se que este fato poderá ocorrer em maiores proporções quando os enfermeiros não possuírem a habilidade clínica para avaliar a ferida.

Identificar a característica do tecido presente na ferida, por meio de sua observação é outro indicador da evolução do processo de cicatrização. Esse método exige do observador conhecimento da fisiologia da cicatrização para identificar as diversas fases desse processo. Alguns autores ${ }^{(1,16,20)}$ correlacionam a coloração do tecido presente na ferida com a fase de cicatrização ou com fatores que podem retardar esse processo.

Há relato que pouca atenção tem sido dada à pele ao redor da ferida, ocasionando problemas como traumas na pele por remoção freqüente de fitas adesivas e alergias aos produtos que recobrem a ferida, manifestados por vermelhidão, bolha e até descamação ${ }^{(18)}$.

$\mathrm{O}$ tecido ao redor da ferida costuma ser o primeiro indicador de lesão nos tecidos. O eritema ao redor da pele é o sinal mais freqüente de lesão tissular e pode indicar injúria por pressão ou fricção. A área ao redor da ferida deve ser avaliada em até quatro centímetros da margem ${ }^{(15)}$.

A característica do exsudato presente nas feridas é um importante indicador que auxilia no diagnóstico clínico de infecção e escolha de terapias tópicas a serem utilizadas. As feridas infectadas têm um retardo do processo de cicatrização e devem ser tratadas devidamente. No processo de cicatrização de feridas, os fluidos contêm enzimas e fatores de crescimento que auxiliam na reparação tecidual e podem ser serosos e sanguinolentos. O exsudato seroso possui coloração amarelo-clara e o sanguinolento apresenta coloração avermelhada. Nas feridas agudas, a presença de exsudato na incisão é normal durante as primeiras 48 a 72 horas. Depois desse período, a presença de exsudato é sinal de prejuízo à cicatrização. A quantidade do exsudato deve diminuir progressivamente na ferida, se esta receber terapia apropriada, 
e pode ser medida usando-se julgamento clínico para avaliar sua distribuição na cobertura ${ }^{(21)}$.

A equipe de enfermagem ao realizar o curativo da ferida deve evitar o traumatismo do tecido de granulação, pois este acarreta sangramento das células e retarda o processo de cicatrização. Os profissionais devem saber diferenciar o exsudato de aspecto seroso do purulento, realizar registros fidedignos e também estar atento para detectar alterações e proporcionar uma adequada intervenção.

\section{CONCLUSÃO}

Com base nos resultados obtidos observou-se que na análise do instrumento, pelos juízes, $71,1 \%$ dos itens obtiveram respostas afirmativas. Sugerindo que o instrumento foi compreendido e pode ser aperfeiçoado com as sugestões manifestadas por eles e acatadas pelas pesquisadoras. Apesar do coeficiente Kappa Múltiplo indicar ausência de concordância e o teste qui-quadrado de Cochran $(p<0,001)$ mostrar diferença no número de respostas afirmativas e negativas entre os juízes, o total de respostas positivas continuou maior, indicando que os juízes compreenderam a maioria dos itens.

Acredita-se que o sistema de classificação por profundidade parece ser mais abrangente que os demais. $\mathrm{Na}$ avaliação da extensão da ferida e do tecido presente no leito da ferida uma das avaliações, em cada um deles, apresentou coeficiente regular reforçando a necessidade de estabelecer melhor os critérios que determinam pontos de referência para a medição, e que os enfermeiros podem apresentar percepções distintas na descrição clínica da característica do tecido. Nas características da pele ao redor da ferida edema, hiperemia, dor e outras alterações apresentaram níveis de concordância regular em pelo menos uma das avaliações e, o aspecto do exsudato e odor nas duas avaliações.

A elaboração de protocolos de avaliação de feridas e treinamentos para os enfermeiros deve ser realizada para incorporar a descrição clínica da ferida como parâmetros mensuráveis.

Sugere-se a aplicação do instrumento pelos profissionais, pois acredita-se na praticidade de seu preenchimento.

\section{REFERÊNCIAS}

1. Flanagan M. Uma estrutura prática para a determinação de ferimentos. 1: Fisiologia. Nursing (Lisboa). 1997; (116): 11-8.

2. Figueredo AM. Ética e cuidado: perspectivas do enfermeiro no tratamento das lesões cutâneas para o próximo milênio. Nursing (São Paulo). 2000; 3(22): 21-5.
3. Healey F. Classificação das úlceras de pressão II. Nursing (Lisboa). 1997; (109): 16-20.

4. Cooper DM. Wound assessment and evaluation of healing. In: Bryant RA. Acute and chronic wounds: nursing management. Philadelphia: Mosby Year Book; c1992. p. 69-90.

5. Sussman C. Assessment of the skin and wound. In: Sussman C, Bates-Jensen BM, editors. Wound care: a collaborative practice manual for physical therapists and nurses. Gaithersburg, Md. : Aspen Publishers; 1998. p. 49-82.

6. Bajay HM, Araújo IEM. Registro da evolução de feridas: elaboração de um instrumento. Rev Gaúch Enferm. 2003; 24 (2): 196-208.

7. Polit DF, Hungler BP. Fundamentos de pesquisa em enfermagem. 3a ed. Porto Alegre: Artes Médicas; 1995.

8. Contandriopoulos AP, Champanhe F, Potvin L, Denis J, Boyle P. Saber preparar uma pesquisa. 3a ed. São Paulo: Hucitec-Abrasco; 1999. cap. 3, p. 57-120.

9. Burns N, Grove SK. The practice of nursing research: conduct, critique, utilization. 3rd ed. Philadelphia: Saunders; c1997. chap. 13, p.319-41.

10. McGibbon G. How to make a questionnaire work. Nurs Times. 1997; 93(23): 46-8.

11. 11.LoBiondo-Wood G, Haber J. Nursing research: methods, critical appraisal and utilization. 4th ed. St Louis: Mosby, c1998. chap.13, p. 327-50.

12. Perroca MG, Gaidzinski RR. Sistema de classificação de pacientes: construção e validade de um instrumento. Rev Esc Enfermagem USP. 1998; 32(2): 153-68.

13. Sawada NO, Galvão CM, Mendes IAC, Coleta JAD. Invasão do território e espaço pessoal do paciente hospitalizado: adaptação de instrumento de medida para a cultura brasileira. Rev Latinoam Enfermagem. 1998; 6(1): 5-10.

14. Fleiss JL. Statistical methods for rates and proportions. 2nd ed. New York: Wiley; c1981.

15. Sussman C, Bates-Jensen BM. Wound care: a collaborative practice manual for physical therapists and nurses. Gaithersburg, Md.: Aspen Publishers; 1998. chap. 5, p.103-23.

16. Marques RR. Avaliação da ferida. In: Gogia PP. Feridas: tratamento e cicatrização. Rio de Janeiro: Revinter; 2003. p. 11-21.

17. Young T. Classificação das úlceras de pressão. Nursing (Lisboa). 1997;(107): 21-5.

18. Dealey C. Cuidando de feridas: um guia para enfermeiras. São Paulo: Atheneu; 1996.

19. Sussman C. Wound measurement. In: Sussman C, BatesJensen BM, editors. Wound care: a collaborative practice manual for physical therapists and nurses. Gaithersburg, Md.: Aspen Publishers; 1998. p.83-102.

20. Cuzzell J, Krasner D. Curatives. In: Gogia PP. Feridas: tratamento e cicatrização. Rio de Janeiro: Revinter; 2003. p. 103-14.

21. Bates-Jensen BM. Management of exsudate and infection. In: Sussman C, Bates-Jensen BM, editors. Wound care: a collaborative practice manual for physical therapists and nurses. Gaithersburg, Md.: Aspen Publishers; 1998. p.159-77. 